\title{
Algorithm for Robust Stability of Delayed Multi-Degree-of-Freedom Systems
}

\author{
Daniel Bachrathy, Marta Janka Reith, Gabor Stepan
}

\begin{abstract}
Computation of the stability limits of systems with time delay is essential in many research and industrial applications. Most of the computational methods consider the exact model of the system, and do not take into account the uncertainties. However, the stability charts are highly sensitive to the change of some input parameters, especially to time-delays. An algorithm has been developed to determine the robust stability limits of delayed dynamical systems, which is not sensitive to the fluctuations of selected parameters in the dynamic system. The algorithm is combined with the efficient Multi-Dimensional Bisection Method. The singledegree-of-freedom delayed oscillator is investigated first and the resultant robust stability limits are compared to the derived analytical results. For multi-degree-offreedom systems, the system of equations of the robust stability limits are modified with the aim to reduce the computational complexity. The method is tested for the 2 -cutter turning system with process damping.
\end{abstract}

\section{Introduction}

The determination of the stability of dynamical systems with time delay is of high importance for numerous industrial and research applications. Some of the most

\footnotetext{
Daniel Bachrathy

Department of Applied Mechanics, BME, H-1111 Budapest, Múegyetem rkp. 5., Hungary, e-mail: e-mail: bachrathy@mm.bme.hu

Marta Janka Reith

Department of Applied Mechanics, BME, H-1111 Budapest, Múegyetem rkp. 5., Hungary, e-mail: e-mail: reith@mm.bme.hu

Gabor Stepan

Department of Applied Mechanics, BME, H-1111 Budapest, Múegyetem rkp. 5., Hungary, e-mail: e-mail: stepan@mm.bme.hu
} 
representative examples are cutting processes [25, 26, 23, 12], wheel shimmy [24], traffic jams [19] and human balancing [11].

One of the most important qualitative properties of dynamical systems is the stability of the equilibrium or the periodic motion. Stability is usually visualized in the form of so-called stability charts, which show the stability boundaries separating those parameter domains for which the system is stable or unstable.

There often exist problems, especially in the field of machining operations at low spindle speeds, where the computation of the stability boundaries requires very high computational effort and unnecessarily high resolution. This is caused by the dense and sharp line segments of the stability boundaries (so-called lobe structure), as it is visible for example in Fig.1 [27]. In case of time-domain computations [12, 13, 27], a high degree of discretization is required for being able to obtain appropriate results. For instance, in frequency domain computations $[6,1,5]$ for cutting processes, the lobe structure becomes dense for low spindle speed values (see Fig.1), which requires high resolution of the stability chart and a continuously growing resolution in the chatter frequency parameter. However, these accurate boundaries are of no interest from practical point of view, thus the computation of the lower envelope of the lobe curves only might be sufficient.

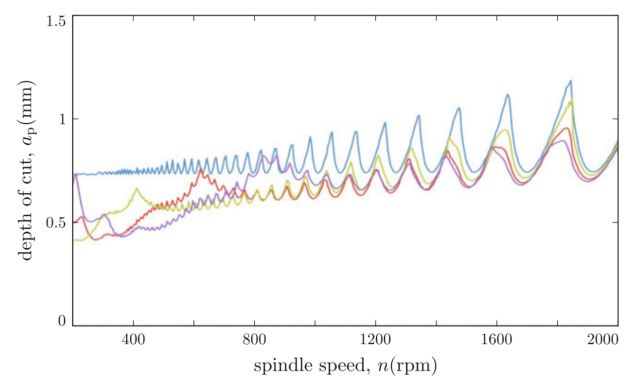

Fig. 1 Stability boundaries in the low spindle speed range for tool geometry optimization presented in [27]

Traditional computational methods consider exact models of the corresponding system and they do not take into account the uncertainties of the input parameters, in spite of the fact, that the results of these computations are highly sensitive to changes of some input parameters like the natural frequencies and time-delays. In practice, these system parameters can only be determined with a limited accuracy.

The parameter uncertainty can be analysed based on the stability radius [17], which considers the perturbation of the elements of the system matrix. In [9] complex perturbation is applied, while [20] considers real valued perturbation. This stability radius method was also applied for time delayed systems in $[16,18]$.

In the present study, a different perturbation method is introduced in order to determine the robust stability limit. The presented algorithm is based on the direct perturbation of the time-delay parameter, only. The main steps are presented based on 
the well-known mathematical model of the delayed oscillator. The resultant robust stability limit is obtained in the form of the lower envelope of the lobe structure. In order to decrease the computational time even further, the method is combined with an efficient numerical root-finding algorithm, the so-called Multi-Dimensional Bisection Method (MDBM) [4, 2]. These robust stability curves are given in analytical form, too. Finally, the presented robust stability computation method is generalized for multi-degree of a freedom systems. The system of equations of the robust stability limits are reformulated to create a more efficient numerical scheme, which is applied to the 2-cutter turning model with process damping [22].

\section{Robust stability of single-degree-of-freedom systems}

In this section, linear time invariant systems are analysed, which can be described by the higher order delay differential equation with a single point delay in the following form:

$$
\sum_{j=0}^{N} l_{j} \frac{\mathrm{d}^{j} x(t)}{\mathrm{d} t^{j}}+\sum_{k=0}^{N-1} r_{k} \frac{\mathrm{d}^{k} x(t-\tau)}{\mathrm{d} t^{k}}=0
$$

where $x$ is the scalar state variable, $N$ is the order of the highest derivative, $l_{j}$ and $r_{k}$ are constant parameters and $\tau$ is the point delay. Note, that we do not deal with neutral equations, hence the highest order derivative term is not delayed.

The robust stability computation algorithm is presented along the model of the second order delayed oscillator (see [10]) given by the governing equation:

$$
\ddot{x}(t)+\kappa \dot{x}(t)+\delta x(t)-b x(t-\tau)=0,
$$

where the time delay is $\tau=2 \pi$. Equation (2) is obtained from Eq.(1) with $N=2$, $\mathbf{I}=[\boldsymbol{\delta}, \kappa, 1]$ and $\mathbf{r}=[b, 0]$.

The corresponding stability charts are computed with the help of the D-subdivision method (for detailed description see [23]) - main steps are summarized below. The characteristic function $D$ of Eq.(1) can be found by substituting the trial function $x(t)=A e^{\lambda t}$ into Eq.(1):

$$
D=\sum_{j=0}^{N} l_{j} \lambda^{j}+\sum_{k=0}^{N-1} r_{k} \lambda^{k} \mathrm{e}^{-\lambda \tau},
$$

which yields the following characteristic function for the delayed oscillator:

$$
D=\lambda^{2}+\kappa \lambda+\delta-b e^{-\lambda \tau} .
$$

The stability boundaries can be determined by substituting the critical value of the characteristic root $\lambda=\mathrm{i} \omega_{\mathrm{c}}$. The co-dimension 2 problem is defined by the real and imaginary parts of the characteristic equation in the parameter space of $\left(\mathbf{l}, \mathbf{r}, \omega_{\mathrm{c}}\right)$ : 


$$
\begin{aligned}
& \Re\left(D\left(\mathbf{l}, \mathbf{r}, \omega_{\mathrm{c}}\right)\right)=0 \\
& \mathfrak{I}\left(D\left(\mathbf{l}, \mathbf{r}, \omega_{\mathrm{c}}\right)\right)=0 .
\end{aligned}
$$

The Multi-Dimensional Bisection Method (MDBM) [4, 2] is designed specifically for these types of root-finding problems, since it is able to find the submanifolds of the roots of a system of non-linear equations in arbitrary parameter dimension and co-dimension. This robust technique can be used for the determination of multiple boundary curves and it can even find closed curves of stable and unstable islands in the stability chart automatically.

For Eq. (2) the free parameters are $\delta, b$ and $\omega_{\mathrm{c}}$, while $\kappa$ is usually considered to be a constant representing damping. The roots of (5) and (6) are determined by MDBM for the test case and are presented in Fig. 2 for $\kappa=0.2$. In the top view (right panel of Fig. 2) the resultant boundary curves form the border of the shaded stable area.
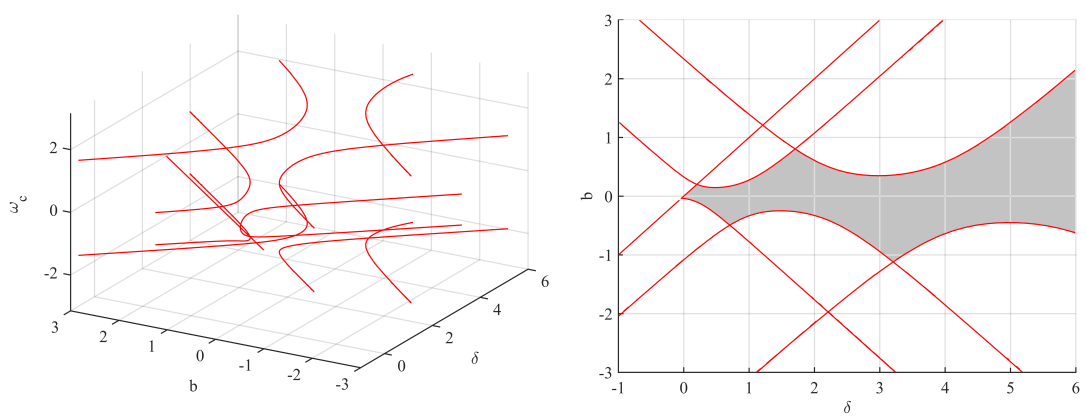

Fig. 2 Resulting curves for the delayed oscillator in the space of $\delta, b$ and $\omega_{\mathrm{c}}$ (left panel) and its top view (right panel), where the stable area is shaded for $\kappa=0.2$

\subsection{Parameter uncertainty}

Equation (2) can be considered as a dimensionless form of the governing equation of a mass-spring system with delayed control. The above computation method considers the exact model of this mechanical system and it does not take into account the uncertainty of the input parameters, like eigenfrequency and feed-back delay. These parameters influence the dimensionless time delay, which is the main source of instability. To represent the effect of uncertainty in the delay parameter $\tau$, numerous stability boundary curves were calculated for a set of time delays in the range $[0,4 \pi]$. These curves are plotted in Fig. 3. The intersection of the stable areas can be used as an approximation of the robust stability region (grey area in the right panel of Fig.3). 

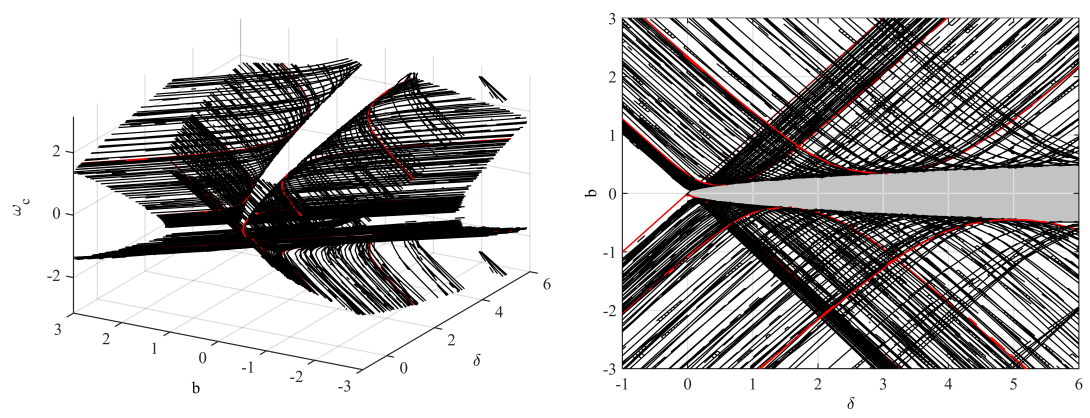

Fig. 3 Resulting curves for the delayed oscillator in the space of $\delta, b$ and $\omega_{\mathrm{c}}$ (left panel) for different discrete $\tau$ values in the range $[0,4 \pi]$ (black lines) where the boundary curves from Fig. 2 are denoted by red thick lines. In the top view (right panel), the approximated robust stable area is shaded for $\kappa=0.2$.

\subsection{Robust stability limit}

The exact robust stability limit has to be computed for continuous time delay variation, however, the computation of the resultant boundaries presented in Fig. 3 was already time consuming, even for only 30 different $\tau$ values. To overcome this problem, a new idea was shown in [3], according to which the set of boundary curves can be connected to a surface by means of an additional parameter, which is defined as the regenerative phase shift parameter

$$
\Phi:=\bmod \left(\tau \omega_{\mathrm{c}}, 2 \pi\right) .
$$

This parameter has to be treated as an independent extra (time-delay perturbation) parameter in the exponential terms, only. Consequently, the set of parameters for the characteristic function is extended by one and it yields:

$$
D=\sum_{j=0}^{N} l_{j} \lambda^{j}+\sum_{k=0}^{N-1} r_{k} \lambda^{k} \mathrm{e}^{-\mathrm{i} \Phi}
$$

For the delayed oscillator, at the stability limit, the characteristic function is given in the form:

$$
D=-\omega_{\mathrm{c}}^{2}+\mathrm{i} \kappa \omega_{\mathrm{c}}+\delta-b e^{-\mathrm{i} \Phi} .
$$

The application of MDBM is essential to solve the resultant co-dimension 2 problem in the extended parameter space. The resultant surface for Eq.(9) is plotted in Fig. 4.

The robust stability limit is defined by the envelope of this surface, where the surface is parallel to the $\omega_{\mathrm{c}}$ axis in the 3D-representation in Fig. 4. It was found, that in the vicinity of the parameter points along the envelope, the real part of the 

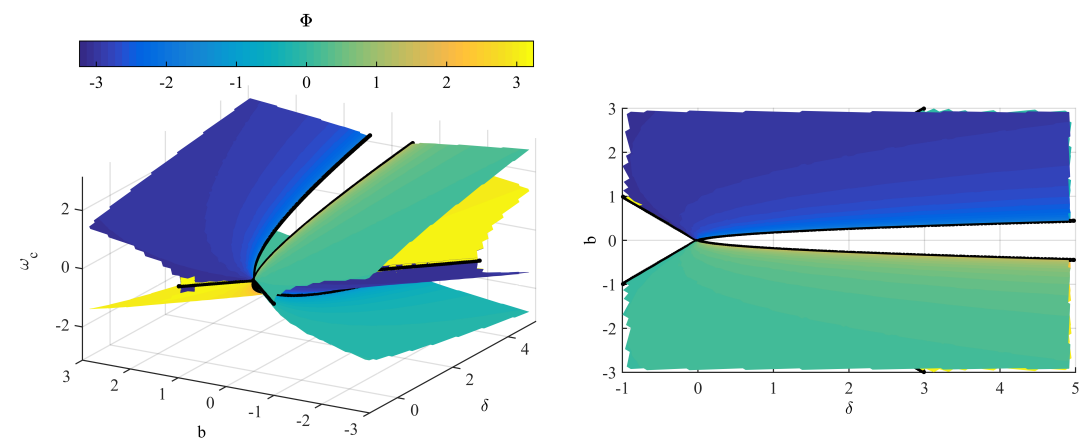

Fig. 4 Surface of the connected stability boundaries of the delayed oscillator in the space of $\delta, b, \omega_{\mathrm{c}}$ and $\Phi$ (left panel) and its top view (right panel). The envelope of the surface forms the robust stability limit given with thick black lines for $\kappa=0.2$.

critical roots $\lambda$ of the characteristic equation (4) does not change as a function of the perturbation parameter. This condition can be described as follows:

$$
\Re\left(\frac{\partial \lambda}{\partial \Phi}\right)=0 \text {. }
$$

The left hand side of Eq.(10) can be determined by the implicit derivation of the characteristic equation [23], which results:

$$
\begin{gathered}
\frac{\mathrm{d} D(\lambda)}{\mathrm{d} \Phi}=0 \\
\frac{\partial D}{\partial \lambda} \frac{\partial \lambda}{\partial \Phi}+\frac{\partial D}{\partial \Phi}=0 .
\end{gathered}
$$

The extra condition (10) necessary for the computation of the robust stability limit can be written with the help of (12) as follows:

$$
\Re\left(\frac{\partial \lambda}{\partial \Phi}\right)=\Re\left(-\frac{\frac{\partial D}{\partial \Phi}}{\frac{\partial D}{\partial \lambda}}\right)=0 .
$$

In order to eliminate the division during the numerical implementation of Eq.(13), it is favourable to use the following rearranged form

$$
\mathfrak{I}\left(\frac{\partial D}{\partial \omega_{\mathrm{c}}} \overline{\frac{\partial D}{\partial \Phi}}\right)=0
$$

in which the critical value $\lambda=\mathrm{i} \omega_{\mathrm{c}}$ is considered and the bar denotes complex conjugate. This extra equation leads to: 


$$
\Re\left(\left(\sum_{j=1}^{N} l_{j} \mathrm{ji}\left(\mathrm{i} \omega_{\mathrm{c}}\right)^{j-1}+\sum_{k=1}^{N-1} r_{k} k \mathrm{i}\left(\mathrm{i} \omega_{\mathrm{c}}\right)^{k-1} \mathrm{e}^{-\mathrm{i} \Phi}\right) \overline{\left(\sum_{k=0}^{N-1} r_{k}\left(\mathrm{i} \omega_{\mathrm{c}}\right)^{k} \mathrm{e}^{-\mathrm{i} \Phi}\right)}\right)=0 .
$$

Note, that the last term in Eq.(15) was already computed during the evaluation of Eq.(8), which can be reused to decrease the computational time. For the delayed oscillator, the extra condition yields

$$
\Re\left(\left(-2 \omega_{\mathrm{c}}+\mathrm{i} \kappa\right)\left(b e^{-\mathrm{i} \Phi}\right)\right)=0 .
$$

Figure 5 shows the traditional stability chart and the robust stability limit of the delayed oscillator, which is determined by MDBM as a co-dimension 3 problem formed by (5), (6) and (16) in the 4 dimensional parameter space $\left(\delta, b, \omega_{\mathrm{c}}, \Phi\right)$.

For 153 grid points along each parameter dimension, the computational time of the traditional stability limit curves was 3.5 s (Matlab 2014b; Intel Core i74710HQM CPU 2.50GHz, 16GB Memory). Thus, the set of stability curves in Fig. 3 was computed in approximately $105 \mathrm{~s}$, while the robust stability limit in Fig. 5 was computed in $4.1 \mathrm{~s}$, only. The computational effort for the robust stability limit is comparable with the time consumed for the computation of the traditional stability limit. Furthermore, the robust stability computation scheme is not only orders of magnitude faster than the approximating method applied for a discrete set of time delays, but it also delivers qualitatively better results in the form of continuous boundary lines, while the results in Fig. 3 present only segmented boundaries.
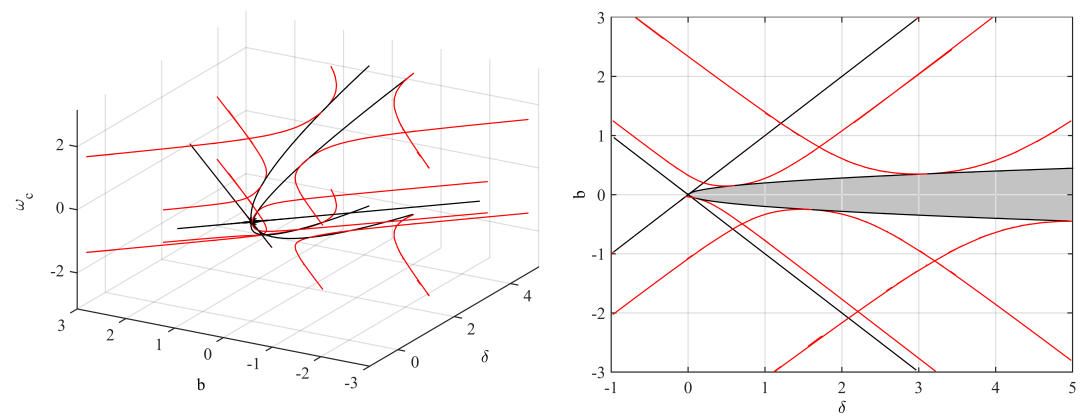

Fig. 5 Stability boundaries of the exact model of the delayed oscillator (red lines - see Fig.2) and the corresponding robust stability limit given with black lines in the space of $\delta, b, \omega_{\mathrm{c}}$ and $\Phi$ (left panel). In the top view (right panel) the robust stable area is shaded for $\kappa=0.2$. 


\subsection{Analytical results for robust stability of delayed oscillator}

For the delayed oscillator, the system of equations (5),(6) and (13) defining the robust stability limit leads to:

$$
\begin{gathered}
-\omega_{\mathrm{c}}^{2}+\delta-b \cos (\Phi)=0, \\
\kappa \omega_{\mathrm{c}}+b \sin (\Phi)=0, \\
-b \kappa \sin (\Phi)-2 b \omega_{\mathrm{c}} \cos (\Phi)=0 .
\end{gathered}
$$

This system can be solved analytically. The first set of results is

$$
\begin{gathered}
b=d \quad \omega_{\mathrm{c}}=0 \quad \Phi=0, \\
b=-d \quad \omega_{\mathrm{c}}=0 \quad \Phi= \pm \pi
\end{gathered}
$$

which are straight robust stability lines corresponding to fold-type bifurcations, while the second set of solutions is:

$$
\begin{gathered}
b= \pm \kappa \sqrt{\delta-\kappa^{2} / 4}, \\
\omega_{\mathrm{c}}= \pm \sqrt{\delta-\kappa^{2} / 2}, \\
\Phi= \pm \operatorname{acos}\left(\frac{\kappa}{4 \delta-\kappa^{2}}\right),
\end{gathered}
$$

which represent the robust stability curves for Hopf-type bifurcations in case of $\delta>\kappa^{2} / 2$.

\section{Robust stability of multi-degree-of-freedom systems}

Let us generalize Eq.(1) for multi-degree-of-freedom systems, which yields the following matrix equation:

$$
\sum_{j=0}^{N} \mathbf{L}_{j} \frac{\mathrm{d}^{j} \mathbf{x}(t)}{\mathrm{d} t^{j}}+\sum_{k=0}^{N-1} \mathbf{R}_{k} \frac{\mathrm{d}^{k} \mathbf{x}(t-\tau)}{\mathrm{d} t^{k}}=0,
$$

where $\mathbf{x} \in \mathbb{R}^{n}, N$ is the order of the highest derivative, $\mathbf{L}_{j}$ and $\mathbf{R}_{k}$ are coefficient matrices and $\tau$ is the point delay. The characteristic equation of Eq.(25) is determined based on the determinant 


$$
D:=\operatorname{det}\left(\sum_{j=0}^{N} \mathbf{L}_{j} \lambda^{j}+\sum_{k=0}^{N-1} \mathbf{R}_{k} \lambda^{k} \mathrm{e}^{-\lambda \tau}\right)=0
$$

using the trial solution $\mathbf{x}(t)=\mathbf{A} e^{\lambda t}$. Since Eq.(26) defines the characteristic equation, all steps described in Section 2 can be performed in order to obtain the robust stability limit. First, the regenerative phase shift parameter is introduced in the exponential terms based on Eq.(7), then the system of equations Eq.(5) and (6) together with the extra condition Eq.(14) are solved by means of MDBM.

The derivative terms in Eq.(14) can be approximated by a finite difference method. This approximation is generally appropriate for numerical schemes. However, it can be determined in closed form, too. Based on this closed form equation, a rearranged form of Eq.(14) is created in order to make the computations more efficient.

\subsection{Optimized numerical solution}

In the extra condition for the robust stability computation given with Eq.(14), the derivative of a determinant must be computed. The closed form solution is provided by Jacobi's formula [14]:

$$
\frac{\mathrm{d}}{\mathrm{d} x} \operatorname{det}(\mathbf{G})=\operatorname{tr}\left(\operatorname{adj}(\mathbf{G}) \frac{\mathrm{d} \mathbf{G}}{\mathrm{d} x}\right),
$$

where $\operatorname{tr}()$ denotes the trace and $\operatorname{adj}()$ is the adjugate matrix (the transpose of the cofactor matrix). Let us define the argument of the determinant of Eq.(26) as

$$
\mathbf{G}=\sum_{j=0}^{N} \mathbf{L}_{j} \lambda^{j}+\sum_{k=0}^{N-1} \mathbf{R}_{k} \lambda^{k} \mathrm{e}^{-\lambda \tau} .
$$

The computational effort for the adjugate matrix can be high, however, in our case, special conditions can be applied. Matrix $\mathbf{G}$ is singular due to Eq.(26), hence $\operatorname{rank}(\mathbf{G})=n-1$. If this holds, the $\operatorname{rank}(\operatorname{adj}(\mathbf{G}))=1$, because the image of $\operatorname{adj}(\mathbf{G})$ is contained in the kernel of $\mathbf{G}$ that has dimension 1 by rank-nullity [15]. This means that the adjugate matrix can be expressed by

$$
\operatorname{adj}(\mathbf{G})=C \mathbf{e}_{0} \circ \mathbf{e}_{0}^{\top},
$$

where $\circ$ denotes the vector direct product or dyadic product and $\mathbf{e}_{0}$ is the eigenvector belonging to the singular eigenvalue $(\mu=0)$ of matrix $\mathbf{G}$. Note, that the constant $C$ depends on the non-singular eigenvalues of $\mathbf{G}$, however, it is not relevant in the further steps of the derivation since it will be cancelled. Equation (29) is valid if the multiplicity of the critical characteristic root is equal to one, otherwise $\operatorname{adj}(\mathbf{G})=\mathbf{0}$ holds. The probability of this situation is very low, especially during the numerical analysis, thus these special cases are not treated here. 
The computation of the only eigenvector $\mathbf{e}_{0}$ can be performed efficiently, e.g. with the inbuilt function eigs in Matlab. Alternatively, the simple text-book-calculation can be used directly if we set the first element of $\mathbf{e}_{0}$ equal to 1 , which leads to

$$
\mathbf{e}_{0}=\left[1, \mathbf{G}_{(2: n) \times(2: n)}^{-1} \mathbf{G}_{(1) \times(2: n)}\right]^{\top} .
$$

For very special parameter sets $\left(e_{0,1} \approx 0\right)$, this could result a poorly conditioned problem. If we consider the special form of the adjugate matrix as in Eq.(29) and the fact that the trace of a dyadic product is the same as the dot product, then Eq.(27) can be reformulated as:

$$
\frac{\mathrm{d}}{\mathrm{d} x} \operatorname{det}(\mathbf{G})=\mathbf{e}_{0}^{\top} \frac{\mathrm{d} \mathbf{G}}{\mathrm{d} x} \mathbf{e}_{0} .
$$

The final form of Eq.(14) for multi-degree-of-freedom systems is

$$
\mathfrak{I}\left(\left(\mathbf{e}_{0}^{\top} \frac{\partial \mathbf{G}}{\partial \omega_{\mathrm{c}}} \mathbf{e}_{0}\right) \overline{\left(\mathbf{e}_{0}^{\top} \frac{\partial \mathbf{G}}{\partial \Phi} \mathbf{e}_{0}\right)}\right)=0,
$$

which leads to the following equation after the substitution of Eq.(28) and after performing the derivations:

$$
\begin{aligned}
& \mathfrak{R}\left(\left(\mathbf{e}_{0}^{\top}\left(\sum_{j=1}^{N} \mathbf{L}_{j} j \mathrm{i}\left(\mathrm{i} \omega_{\mathrm{c}}\right)^{j-1}+\sum_{k=1}^{N-1} \mathbf{R}_{k} k \mathrm{i}\left(\mathrm{i} \omega_{\mathrm{c}}\right)^{k-1} \mathrm{e}^{-\mathrm{i} \Phi}\right) \mathbf{e}_{0}\right)\right. \\
& \left.\overline{\left(\mathbf{e}_{0}^{\top}\left(\sum_{k=0}^{N-1} \mathbf{R}_{k}\left(\mathrm{i} \omega_{\mathrm{c}}\right)^{k} \mathrm{e}^{-\mathrm{i} \Phi}\right) \mathbf{e}_{0}\right)}\right)=0 .
\end{aligned}
$$

Note, that this formula is only valid at the boundary of the robust stability area. If we are off the robust stability curve, then this equation is an approximation only, because $\mathbf{G}$ is not singular. For these off-boundary points $\mathbf{e}_{0}$ can be approximated by Eq.(30) or by the eigenvector belonging to the eigenvalue with the smallest magnitude.

If we decide to compute the eigenvector $\mathbf{e}_{0}$ by solving the eigenvalue-eigenvector problem, then the corresponding eigenvalue $\mu_{0}$ with the smallest magnitude is also obtained as a side result, which can be used instead of the determinant in Eq.(26), because $\operatorname{det}(\mathbf{G})=\prod_{m=1}^{n} \mu_{m}=0$ if and only if $\mu_{0}=0$.

The above presented robust stability computation method is applied for a twocutter turning system with process damping. 


\subsection{Robust stability limit of the two-cutter turning with process damping}

In order to present the efficiency of the above presented robust stability computation method, it is applied for the test case of the two-cutter turning process with process damping. The model and the corresponding equation of motion of a two-cutter turning system is given in [21, 22] where the dynamics of the turret is also modelled. The simplified mechanical model is shown in Fig.6. If the effect of process damping $[26,8,7]$ is taken into account, too, then an extra damping coefficient appears, which is proportional to the time delay $\tau$ and thus the governing equation can be written in the form:

$$
\mathbf{M} \ddot{\mathbf{x}}(t)+\left(\mathbf{C}+c_{\mathrm{p}} \tau \mathbf{E}\right) \dot{\mathbf{x}}(t)+\mathbf{K} \mathbf{x}(t)=k_{\mathrm{w}}(\mathbf{L x}(t-\tau)-\mathbf{E x}(t)) .
$$

Here, $\mathbf{x}$ is the vector of the position coordinates of the tools and the turret (see Fig.6), $c_{\mathrm{p}}$ denotes the process damping coefficient, $k_{\mathrm{w}}$ is the cutting coefficient. The coefficient matrices in Eq.(34) are given in Tab.1.

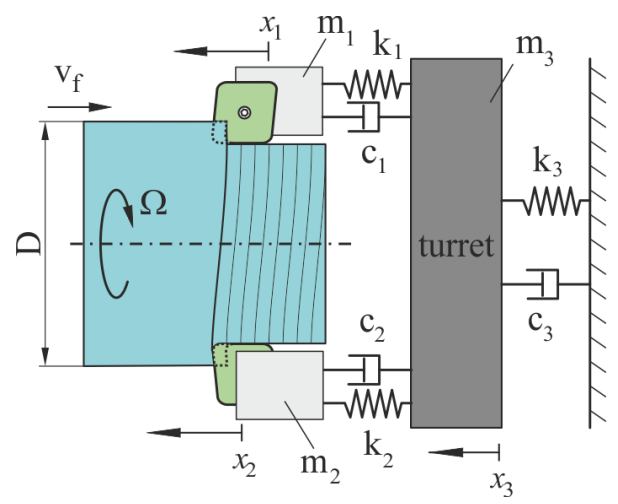

Fig. 6 Schematic representation of the two-cutter turning model. Tool 1 and tool 2 are coupled via the cutting force function, too, due to the surface regeneration effect.

Table 1 The coefficient matrices in the governing equation of the two-cutter turning model

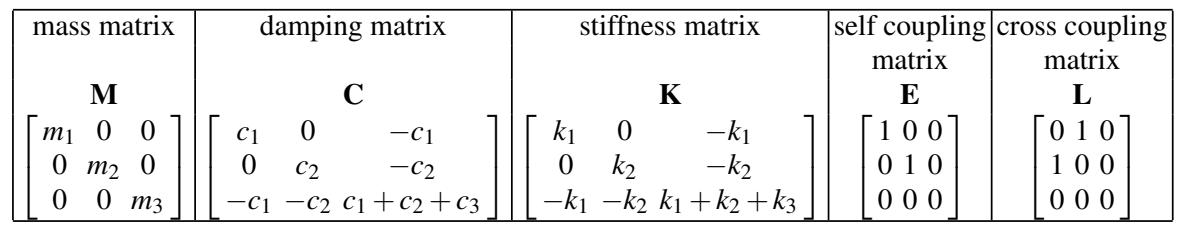

The corresponding characteristic equation for $\lambda=i \omega_{c}$ with the regenerative phase shift parameter $\Phi$ is given by: 


$$
\operatorname{det}\left(-\omega_{\mathrm{c}}^{2} \mathbf{M}+\mathrm{i} \omega_{\mathrm{c}} \mathbf{C}+\mathrm{i} c_{\mathrm{p}} \tau \omega_{\mathrm{c}} \mathbf{E}+\mathbf{K}-k_{\mathrm{w}}\left(\mathbf{L} e^{-\mathrm{i} \Phi}-\mathbf{E}\right)\right)=0,
$$

Note, that the term $\tau \omega_{\mathrm{c}}$ is replaced by $\Phi$ in the exponential term only, while in the damping term it is left as it is.

The resulting stability boundary curves (red) and the robust stability limit (black) are shown in Fig.7 in the plane of the spindle speed $\Omega=2 \pi / \tau$ and the cutting coefficient $k_{\mathrm{w}}$.

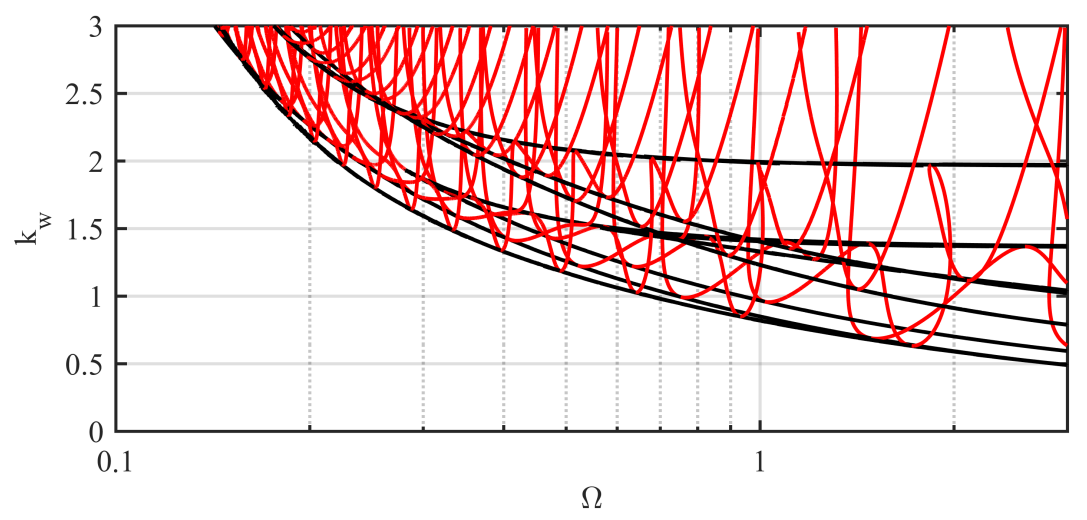

Fig. 7 Stability boundaries of the two-cutter turning system (red lines) and its robust stability limits (black lines) for the dimensionless parameters: $m_{1}=m_{2}=1, m_{3}=10, c_{1}=c_{2}=0.02, c_{3}=0.2, k_{1}=1, k_{2}=4$, $k_{3}=40, c_{\mathrm{p}}=0.03$. Note, the logarithmic scale of $\Omega$.

In spite of the high resolution of the computed stability chart, the dense stability lobe structure (red curves) becomes more and more inaccurate in the lower spindle speed range. It can be observed in Fig.7 that the robust stability lines (black) are smooth in the whole spindle speed range for the same resolution, while the computational time is in the same range as for the stability computation.

\section{Conclusion}

In the present study, we have developed a method based on parameter perturbation for calculating the robust stability limits of systems of differential equations with point delay. One of the main benefits of the presented method is, that it eliminates the sensitivity of the stability charts to the time delay parameter. Thus it can be efficiently used in cases where some of the input parameters are inaccurate.

In the proposed computation method, an additional perturbation parameter has to be introduced in order to find the resultant robust stability curves, which form the envelopes of the stability boundary lines. This increases the dimension of the parameter space by one, which requires the formulation of an additional condition 
for the robust stability limit curve. Although the set of parameters and the system of equations are extended, the applied Multi-Dimensional Bisection Method can efficiently solve the equations without significant increase of computational time.

Future research is planned to extend this method to dynamical systems with multiple and/or distributed delays.

Acknowledgements The research leading to these results has received funding from the European Research Council under the European Unions Seventh Framework Programme (FP7/2007-2013) ERC Advanced grant agreement No340889. Furthermore, this paper was supported by the Hungarian Scientific Research Fund - OTKA PD-112983 and the Janos Bolyai Research Scholarship of the Hungarian Academy of Sciences.

\section{References}

1. Altintas, Y., Stepan, G., Merdol, D., Dombovari, Z.: Chatter stability of milling in frequency and discrete time domain. CIRP Journal of Manufacturing Science and Technology 1(1), 35-44 (2008). DOI doi:10.1016/j.cirpj.2008.06.003

2. Bachrathy, D.: Multi-dimensional bisection method (mdbm) - fast computation of all the roots of a non-linear function for higher parameter dimension and co-dimension (2012)

3. Bachrathy, D.: Robust stability limit of delayed dynamical systems. Periodica Polytechnica. Engineering. Mechanical Engineering 59(2), 74 (2015)

4. Bachrathy, D., Stepan, G.: Bisection method in higher dimensions and the efficiency number. Periodica polytechnica. Mechanical engineering 56(2), 81-86 (2012)

5. Bachrathy, D., Stepan, G.: Improved prediction of stability lobes with extended multi frequency solution. CIRP Annals - Manufacturing Technology pp. - (2013). DOI 10.1016/j.cirp.2013.03.085

6. Budak, E., Altintas, Y.: Analytical prediction of chatter stability conditions for multi-degree of systems in milling. Transactions of ASME Journal of Dynamic Systems Measurement and Control 120, 22-30 (1998)

7. Budak, E., Tunc, L.T.: Identification and modeling of process damping in turning and milling using a new approach. CIRP Annals-Manufacturing Technology 59(1), 403-408 (2010)

8. Eynian, M., Altintas, Y.: Chatter stability of general turning operations with process damping. Journal of Manufacturing Science and Engineering 131(4), 10 (2009). DOI 10.1115/1.3159047

9. Hinrichsen, D., Pritchard, A.J.: Stability radius for structured perturbations and the algebraic riccati eauation. Systems \& Control Letters 8, 105 - 113 (1986)

10. Hsu, C.S., Bhatt, S.: Stability charts for second-order dynamical systems with time lag. Journal of applied mechanics 33(1), 119-124 (1966)

11. Insperger, T., Milton, J., Stepan, G.: Acceleration feedback improves balancing against reflex delay. Journal of the Royal Society Interface 10(79), 20120,763 (2012). DOI 10.1098/rsif.2012.0763 1742-5662

12. Insperger, T., Stepan, G.: Semi-Discretization for Time-Delay Systems, 1 edn. Applied Mathematical Sciences. Springer, 1 (2011)

13. Khasawneh, F.A., Mann, B.P., Butcher, E.A.: Comparison between collocation methods and spectral element approach for the stability of periodic delay systems. In: 9th IFAC Workshop on Time Delay Systems, vol. 9, pp. 69-74 (2010)

14. Magnus, J.R., Neudecker, H., et al.: Matrix differential calculus with applications in statistics and econometrics (1995)

15. Meyer, C.D.: Matrix analysis and applied linear algebra, vol. 2. Siam (2000) 
16. Michiels, W., Fridman, E., Niculescu, S.I.: Robustness assessment via stability radii in delay parameters. Int. J. Robust Nonlinear Control (2008). DOI DOI: 10.1002/rnc.1385

17. Michiels, W., Niculescu, S.I.: Stability and Stabilization of Time-Delay Systems. Society for Industrial and Applied Mathematics, Philadelphia (2007)

18. Michiels, W., Roose, D.: An eigenvalue based approach for the robust stabilization of linear time-delay systems. INT. J. CONTROL 76(7), 678-686 (2003)

19. Orosz, G., Wilson, R.E., Stepan, G.: Traffic jams: dynamics and control. Philosophical Transactions of the Royal Society of London A: Mathematical, Physical and Engineering Sciences 368(1928), 4455-4479 (2010). DOI 10.1098/rsta.2010.0205

20. Qiu, L., Bernhardsson, B., Rantzer, A., Davison, E.J., Young, P.M., Doyle, J.C.: A formula for computation of the real stability radius. IMA Preprint Series \# 1160 (1993)

21. Reith, M.J., Bachrathy, D., Stepan, G.: Comparing stability and dynamic behaviour of different multi-cutter turning models. In: ENOC 2014, Wien,, 494, p. 2 (2014)

22. Reith, M.J., Stepan, G.: Exploitation of non-proportional damping in machine tools for chatter suppression. Journal Mechanism and Machine Theory submitted (2016)

23. Stepan, G.: Retarded Dynamical Systems. Longman, Harlow (1989)

24. Takacs, D.: Dynamics of towed wheels - nonlinear theory and experiments. Ph.D. thesis, Budapest University of Technology and Economics, Department of Applied Mechanics (2011)

25. Tlusty, J., Spacek, L.: Self-excited vibrations on machine tools. Prague, Czech Republic: Nak1 CSAV. [In Czech.] (1954)

26. Tobias, S.A.: Machine Tool Vibration. Blackie and Son, Ltd., London (1965)

27. Zatarain, M., Dombovari, Z.: Stability analysis of milling with irregular pitch tools by the implicit subspace iteration method. International Journal of Dynamics and Control 2(1), 2634 (2014) 